\title{
FATORES DE RISCO PARA INFECÇÃO DE SÍTIO CIRÚRGICO EM PROCEDIMENTOS CIRÚRGICOS CARDÍACOS
}

\author{
RISK FACTORS FOR SURGICAL SITE INFECTION IN \\ CARDIAC SURGICAL PROCEDURES
}

\section{FACTORES DE RIESGO PARA INFECCIÓN DE SITIO QUIRÚRGICO EN PROCEDIMIENTOS QUIRÚRGICOS CARDÍACOS}

\author{
Cláudia Silva Marinho Antunes Barros \\ Ana Lúcia Arcanjo Oliveira Cordeiro² \\ Luana Stela de Araújo Castro ${ }^{3}$ \\ Marimeire Morais Conceição ${ }^{4}$ \\ Márcia Maria Carneiro Oliveira
}

Como citar este artigo: Barros CSMA, Cordeiro ALAO, Castro LSA, Conceição MM, Oliveira MMC. Fatores de risco para infecção de sítio cirúrgico em procedimentos cirúrgicos cardíacos. Rev baiana enferm. (2018);32:e26045.

Objetivo: descrever os fatores de risco para infecção de sítio cirúrgico presentes em pacientes submetidos a procedimentos cirúrgicos cardíacos. Método: estudo de caso-controle, retrospectivo e descritivo. Participaram os pacientes submetidos a procedimentos cirúrgicos cardíacos entre 2011 e 2013 que desenvolveram e não desenvolveram infecção. Os dados foram coletados na Comissão de Controle de Infeção Hospitalar e nos prontuários, entre maio e dezembro de 2014. Resultados: foram estudados 50 pacientes, 19 casos e 31 controles. A ocorrência de infecção foi mais frequente em idosos do sexo masculino. Ambos os grupos apresentaram semelhanças na prevalência dos fatores de risco: tempo de internação pré-operatório superior a 24 horas, classificados no nível III, pelo physical status classification system, eram portadores de hipertensão e de diabetes e houve descontinuidade da antibioticoprofilaxia. Conclusão: os fatores de risco para infecção de sítio cirúrgico em pacientes submetidos a cirurgias cardíacas requerem dos profissionais envolvidos no processo maior controle para reduzir os índices dessas complicações.

Descritores: Infecção da ferida cirúrgica. Fatores de risco. Cirurgia torácica. Enfermagem.

Objective: describe the risk factors for surgical site infection in patients submitted to cardiac surgical procedures. Method: retrospective and descriptive case-control study. The participants were the patients submitted to cardiac surgical procedures between 2011 and 2013 who developed and who did not develop an infection. The data were collected from the Hospital Infection Control Committee and in the patient histories between May and December 2014. Results: fifty patients were studied, being 19 cases and 31 controls. Infection occurred more frequently in male elderly patients. Both groups showed similarities in the prevalence of the following risk factors: length of preoperative

\footnotetext{
Enfermeira. Doutora em Ciências. Professora Adjunta da Escola de Enfermagem da Universidade Federal da Bahia. Salvador, Bahia, Brasil. marinho-claudia@hotmail.com

Enfermeira. Doutora em Enfermagem. Professora Associada da Escola de Enfermagem da Universidade Federal da Bahia. Salvador, Bahia, Brasil.

Enfermeira. Especialista em Enfermagem em Centro Cirúrgico e Central de Material Esterilizado. Enfermeira assistencial da Unidade de Centro Cirúrgico do Hospital Cárdio Pulmonar. Salvador, Bahia, Brasil.

Enfermeira assistencial do Centro Cirúrgico do Hospital Geral Roberto Santos. Salvador, Bahia, Brasil.

Enfermeira. Doutoranda em Medicina e Saúde pela Universidade Federal da Bahia. Professora Assistente da Escola de Enfermagem da Universidade Federal da Bahia. Salvador, Bahia, Brasil.
} 
hospitalization longer than 24 bours, classified under physical status classification system level III. The patients suffered from bypertension and diabetes and the antibiotic prophylaxis was discontinued. Conclusion: the risk factors for surgical site infection in patients submitted to cardiac surgeries demand greater control from the professionals involved in the process with a view to reducing the rates of these complications.

Descriptors: Surgical wound infection. Risk factors. Thoracic surgery. Nursing.

Objetivo: describir los factores de riesgo para infección de sitio quirúrgico en pacientes sometidos a procedimientos quirúrgicos cardiacos. Método: estudio de caso-control, retrospectivo y descriptivo. Participaron pacientes sometidos a procedimientos quirúrgicos cardiacos entre 2011 y 2013 que desarrollaron y no desarrollaron infección. Datos recolectados en la Comisión de Control de Infección Hospitalaria y en registros médicos, entre mayo y diciembre de 2014. Resultados: se estudiaron 50 pacientes, 19 casos y 31 controles. Incidencia de infección más frecuente en ancianos varones. Ambos los grupos presentaron semejanzas en la prevalencia de los factores de riesgo: tiempo de internación preoperatorio superior a 24 horas; clasificados en el nivel III, por el physical status classification system; portadores de hipertensión y diabetes; y en discontinuidad de la antibioticoprofilaxis. Conclusión: los factores de riesgo para infección de sitio quirúrgico en pacientes sometidos a cirugías cardiacas requieren de los profesionales involucrados en el proceso mayor control para reducir los indices de esas complicaciones.

Descriptores: Infección de la herida quirúrgica. Factores de riesgo. Cirugía torácica. Enfermería.

\section{Introdução}

Entre as infecções hospitalares, a infecção de sítio cirúrgico (ISC) está entre as principais Infecções Relacionadas à Assistência à Saúde (IRAS), representando 17\%. Estima-se que ocorrem 240 mil casos por ano nos Estados Unidos da América $^{(1)}$. No Brasil, a ISC ocupa a terceira posição entre todas as IRAS e compreende $14 \%$ a $16 \%$ daquelas encontradas em pacientes hospitalizados $^{(2)}$.

De acordo com o levantamento realizado no Departamento de Tecnologia da Informação a Serviço do SUS (DATASUS), no período compreendido entre 2010 e 2014, o total de cirurgias cardíacas realizadas no Brasil correspondeu a 372.250, com 23.382 óbitos. Na Bahia, mais de 15.000 cirurgias cardíacas foram realizadas com um total de 634 óbitos. Em Salvador foram realizadas 11.910 cirurgias com aproximadamente 500 (4,2\%) óbitos registrados após o procedimento cirúrgico ${ }^{(3)}$. Considerando-se a taxa de ISC descrita para o Brasil, aproximadamente 1.667 (14\%) a 1.905 (16\%) desses pacientes poderiam ter desenvolvido ISC e ainda ter ocorrido entre 70 (14\%) e 80 (16\%) óbitos por ISC e/ou suas consequências ${ }^{(3)}$.

As ISCs ocorrem nos primeiros 30 dias após a cirurgia ou até um ano, no caso de uso de próteses $^{(4)}$. São classificadas em incisional superficial, quando envolve pele e tecido subcutâneo; incisional profunda, quando envolve tecidos moles profundos, como fáscia e/ou músculos; e órgãos/cavidade quando envolve qualquer órgão ou cavidade, denominada mediastinite, em cirurgia cardíaca ${ }^{(4-5)}$.

Os fatores de risco associados ao desenvolvimento da ISC na cirurgia cardíaca estão relacionados com o paciente no período pré-operatório, tais como idade avançada, diabetes mellitus mal controlado, obesidade, uso do tabaco, uso de medicações imunossupressoras e longo tempo de internamento hospitalar no pré-operatório; e aqueles relacionados com o procedimento ou no período perioperatório, que incluem o tempo de cirurgia, tricotomia, hipóxia e hipotermia ${ }^{(6-7)}$.

A literatura aponta que os pacientes expostos a longa hospitalização prévia à cirurgia cardíaca apresentaram maior prevalência de complicações infecciosas no pós-operatório $^{(7)}$. Estudo realizado com 19.333 pacientes submetidos a procedimentos cirúrgicos cardíacos no período de 1995 a 2012, no Canadá, identificou que 2.726 (14\%) contraíram pelo menos uma infecção. Os fatores de risco relacionados ao desenvolvimento desse processo corresponderam a idade avançada, sexo feminino, fração de ejeção baixa, doença vascular periférica e doença vascular cerebral, insuficiência renal, diabetes, insuficiência cardíaca congestiva, 
reabordagens operatórias, procedimentos cardíacos combinados, doença pulmonar obstrutiva crônica e procedimentos de emergência ${ }^{(6)}$.

Os estudos sobre os fatores de risco que contribuem para a ocorrência de ISC podem fornecer subsídios para o planejamento e a adoção de estratégias na prevenção, no controle e no monitoramento dessa infecção, a fim de minimizar sua ocorrência e assegurar os princípios da segurança do paciente cirúrgico.

Nesse sentido, questiona-se: Quais os fatores de risco para ISC presentes em pacientes submetidos a cirurgia cardíaca em um Hospital Universitário?

O estudo teve como objetivo descrever os fatores de risco para ISC presentes em pacientes submetidos a procedimentos cirúrgicos cardíacos.

\section{Método}

Trata-se de um estudo epidemiológico, de caso-controle, retrospectivo e descritivo sobre ISC em pacientes submetidos a procedimentos cirúrgicos cardíacos no período de 2011 a 2013, em um Hospital Universitário, público, federal, geral, de grande porte, que faz parte de um Complexo Hospitalar e Ambulatorial, localizado no estado da Bahia, região Nordeste do Brasil.

A amostra foi de conveniência e foram incluídos inicialmente como população todos os pacientes submetidos a procedimentos cirúrgicos cardíacos, no centro cirúrgico do Hospital Universitário, que tiveram diagnóstico de infecção definido no pós-operatório e o tratamento realizado durante internamento no hospital. Esses casos apresentaram registro confirmatório de ISC pela Comissão de Controle de Infecção Hospitalar do Hospital (CCIH) e seu acompanhamento no período de interesse. Em seguida, para seleção dos pacientes do grupo controle, foram aplicados os critérios de pareamento definidos: pacientes que realizaram a mesma cirurgia, do mesmo sexo, e variação na idade de \pm 5 anos.

O diagnóstico de ISC é baseado em critérios definidos ${ }^{(1)}$ : confirmação da infecção dada pelo médico assistente; uso de indicadores de vigilância, como antibioticoterapia; resultados de exames e culturas; além dos registros médicos e de enfermagem disponíveis nos prontuários dos pacientes.

A coleta de dados foi realizada no período de maio a dezembro de 2014, utilizando-se as bases de dados das unidades da CCIH e do Serviço de Arquivo Médico e Estatística (SAME), prontuários físicos e eletrônicos, considerando as variáveis de interesse relacionadas ao estudo.

Os grupos de variáveis selecionadas para caracterizar o perfil dos pacientes foram relacionadas especialmente aos fatores de risco reconhecidos na literatura para o desenvolvimento de ISC. Assim, foram coletadas variáveis sociodemográficas: idade, sexo, cor/raça, procedência, situação ocupacional; clínicas prévias: hipertensão arterial sistêmica (HAS), diabetes mellitus (DM), cardiopatias, doença respiratória, desnutrição/baixo peso, doença imunológica, insuficiência renal crônica (IRC); e do processo cirúrgico (hospitalização e realização da cirurgia): tempo de internação hospitalar no pré-operatório, classificação da cirurgia (emergência/urgência), tempo cirúrgico, ASA physical status classification system (ASA) e antibioticoprofilaxia.

Este estudo obteve aprovação do Comitê de Ética em Pesquisa (CEP) do Hospital, com Parecer n. 573.351, e atendeu às diretrizes da Resolução n. 466/12 do Conselho Nacional de Saúde, sobre pesquisa envolvendo seres humanos.

Os dados coletados foram digitados em um banco de dados criado para este fim, por meio do software Data Analysis and Statistical (STATA), versão 13. Para análise dos dados foi utilizada a estatística descritiva.

\section{Resultados}

Conforme os Relatórios Anuais da CCIH do hospital, ocorreram, no período de interesse, 1.056 casos de infecções hospitalares (IHs). Destas 114 $(10,8 \%)$ foram ISCs e, dessas últimas, 19 (16,7\%) foram desenvolvidas por pacientes após serem submetidos a procedimentos cirúrgicos cardíacos. No ano de 2011, ocorreram 4 casos; em 2012 foram 10 casos; e em 2013, 5 casos. De acordo com a metodologia proposta, deveriam ser analisados 38 controles, respeitando-se a proporção 
1:2. No entanto, foram analisados 31 , obtendo-se a proporção de 1:1,63 devido à aplicação dos critérios de pareamento e exclusão de casos de pacientes que apresentavam processo infeccioso antes do procedimento cirúrgico, totalizando, assim, 50 pacientes estudados.

A Tabela 1 demonstra que, dos pacientes que desenvolveram ISC, 64,7\% foram do sexo masculino, e a faixa etária mais frequente foi acima de 60 anos (63,2\%). A maioria declarou-se parda, proveniente do interior do estado, aposentado ou exercia outras atividades, como professor, pintor, padeiro, médico veterinário, guia turístico, técnico em administração, lavrador, cozinheiro, comerciante e motorista. Os dados evidenciaram ainda os altos índices de paciente portadores de HAS (78,9\% e 83,9\%) e de DM $(42,1 \%$ e 48,4\%) dentre os casos e os controles, respectivamente. A desnutrição, doença respiratória, doença imunológica e a IRC foram comorbidades menos prevalentes entre os pacientes estudados.

Tabela 1 - Características sociodemográficas e clínicas de pacientes submetidos a procedimentos cirúrgicos cardíacos em um Hospital Universitário. Salvador, Bahia, Brasil - 2015 (N=50)

\begin{tabular}{|c|c|c|}
\hline $\begin{array}{l}\text { Características } \\
\text { Sociodemográficas }\end{array}$ & $\begin{array}{c}\text { Pacientes com Infecção } \\
\text { de Sítio Cirúrgico } \\
\text { CASO }(\mathbf{n}=19)\end{array}$ & $\begin{array}{c}\text { Pacientes sem Infecção de } \\
\text { Sítio Cirúrgico } \\
\text { CONTROLE }(\mathbf{n}=\mathbf{3 1})\end{array}$ \\
\hline \multicolumn{3}{|l|}{ Grupo etário } \\
\hline 30 a 60 anos & $7(36,8 \%)$ & $14(45,2 \%)$ \\
\hline Acima de 60 anos & $12(63,2 \%)$ & $17(54,8 \%)$ \\
\hline \multicolumn{3}{|l|}{ Sexo } \\
\hline Feminino & $8(42,1 \%)$ & $14(45,2 \%)$ \\
\hline Masculino & $11(64,7 \%)$ & $17(54,8 \%)$ \\
\hline \multicolumn{3}{|l|}{ Cor/Raça } \\
\hline Branco & $5(26,3 \%)$ & $5(16,1 \%)$ \\
\hline Preto & $6(31,6 \%)$ & $8(25,8 \%)$ \\
\hline Pardo & $8(42,1 \%)$ & $18(58,1 \%)$ \\
\hline \multicolumn{3}{|l|}{ Procedência } \\
\hline Capital & $9(47,4 \%)$ & $14(45,2 \%)$ \\
\hline Interior & $10(52,6 \%)$ & $17(54,8 \%)$ \\
\hline \multicolumn{3}{|l|}{ Ocupação } \\
\hline Aposentado & $7(36,8 \%)$ & $14(45,2 \%)$ \\
\hline Dona do lar/Doméstica & $4(21,1 \%)$ & $3(9,7 \%)$ \\
\hline Outras atividades & $6(31,6 \%)$ & $13(41,9 \%)$ \\
\hline Desempregado & $2(10,5 \%)$ & - \\
\hline \multicolumn{3}{|l|}{ Clínicas/comorbidades } \\
\hline Hipertensão arterial sistêmica & $15(78,9 \%)$ & $26(83,9 \%)$ \\
\hline Diabetes mellitus & $8(42,1 \%)$ & $15(48,4 \%)$ \\
\hline Doença respiratória & $2(10,5 \%)$ & - \\
\hline Desnutrição/Baixo peso & $5(26,3 \%)$ & $3(9,7 \%)$ \\
\hline Doença imunológica & $1(5,3 \%)$ & - \\
\hline Insuficiência renal crônica & $1(5,3 \%)$ & $1(3,2 \%)$ \\
\hline
\end{tabular}

Fonte: Elaboração própria.

Nota: Sinal convencional utilizado:

- Dado numérico igual a zero não resultante de arredondamento.

A Tabela 2 mostra que todos os pacientes - casos e controles - tiveram tempo de internação pré-operatório maior que 24 horas. O procedimento cirúrgico mais realizado foi a revascularização do miocárdio, eletivo, com duração entre 4 e 8 horas. A maioria dos pacientes foi classificada como ASA III (com doença sistêmica grave, com limitação funcional, mas não 
incapacitante). Prevaleceu o uso da antibioticoprofilaxia em ambos os grupos. A maioria dos participantes, incluindo todos do grupo controle, fez a continuação da antibioticoprofilaxia por 24 horas após a cirurgia. A antibioticoterapia (mais de 24 horas) foi utilizada na maioria desses pacientes após os procedimentos, incluindo todos os pacientes do grupo caso.

Tabela 2 - Características do processo cirúrgico de pacientes submetidos a procedimentos cirúrgicos cardíacos em um Hospital Universitário. Salvador, Bahia, Brasil - 2015 (N=50)

\begin{tabular}{|c|c|c|}
\hline Características do processo cirúrgico & $\begin{array}{c}\text { Pacientes com Infecção } \\
\text { de Sítio Cirúrgico } \\
\text { CASO }(\mathbf{n}=19)\end{array}$ & $\begin{array}{c}\text { Pacientes sem Infecção } \\
\text { de Sítio Cirúrgico } \\
\text { CONTROLE }(\mathbf{n}=31)\end{array}$ \\
\hline \multicolumn{3}{|l|}{ Tempo de internação pré-operatório } \\
\hline Acima de 24 horas & $19(100 \%)$ & $31(100 \%)$ \\
\hline \multicolumn{3}{|l|}{ Procedimento cirúrgico } \\
\hline Revascularização do miocárdio & $13(68,4 \%)$ & $24(77,4 \%)$ \\
\hline Troca/Implante de prótese valvar ou mitral & $5(26,3 \%)$ & $5(19,1 \%)$ \\
\hline Implante de marcapasso & $1(5,3 \%)$ & - \\
\hline Valvuloplastia mitral/aórtica & - & $2(6,45 \%)$ \\
\hline \multicolumn{3}{|l|}{ Emergência/Urgência } \\
\hline $\operatorname{Sim}$ & $2(10,5 \%)$ & - \\
\hline Não & $17(89,5 \%)$ & $31(100 \%)$ \\
\hline \multicolumn{3}{|l|}{ Duração da cirurgia } \\
\hline Até 2 horas & $1(5,3 \%)$ & - \\
\hline 2 a 4 horas & $8(42,1 \%)$ & $11(35,5 \%)$ \\
\hline Mais de 4 a 6 horas & $8(42,1 \%)$ & $18(58 \%)$ \\
\hline Mais de 6 horas & $2(10,5 \%)$ & $2(6,45 \%)$ \\
\hline \multicolumn{3}{|l|}{$\boldsymbol{A S A} \boldsymbol{A}^{*}$} \\
\hline II & $6(31,6 \%)$ & $1(3,2 \%)$ \\
\hline III & $12(63,1 \%)$ & $25(80,6 \%)$ \\
\hline IV & $1(5,3 \%)$ & $5(16,1 \%)$ \\
\hline \multicolumn{3}{|l|}{ Uso de Antibióticos } \\
\hline Antibioticoprofilaxia & $18(94,3 \%)$ & $30(96,8 \%)$ \\
\hline Continuação do antibiótico até 24 horas & $12(63,2 \%)$ & $31(100 \%)$ \\
\hline Antibioticoterapia por mais de 24 horas & $19(100 \%)$ & $21(67,7 \%)$ \\
\hline
\end{tabular}

Fonte: Elaboração própria.

Nota: Sinal convencional utilizado:

- Dado numérico igual a zero não resultante de arredondamento.

*ASA physical status classification system (ASA sistema de classificação do estado físico), adaptado da American Society of Anesthesiologists (Sociedade Americana dos Anestesiologistas) ${ }^{(8)}$.

$A S A$ I: paciente saudável, normal.

$A S A$ II: paciente com uma doença sistêmica leve, sem limitações funcionais importantes.

ASA III: paciente com uma ou mais de uma doença sistêmica moderada ou grave, com limitação funcional importante.

$A S A$ IV: paciente com doença sistêmica grave, com ameaça constante à vida.

$A S A$ V: paciente moribundo, onde não é esperado que sobreviva sem cirurgia.

$A S A$ VI: paciente com morte cerebral declarada cujo órgãos serão removidos para fins de transplantes.

\section{Discussão}

Considerando as características sociodemográficas, destacou-se a presença de ISC em pacientes idosos, com idade maior que 60 anos, e do sexo masculino. Estudo realizado na região Sudeste do Brasil, com 82 pacientes submetidos a cirurgias cardíacas no ano de 2015, encontrou a predominância de ISC em pacientes do sexo masculino e idosos, $75,60 \%$ e 51,21\%, respectivamente ${ }^{(9)}$, corroborando os achados deste estudo. Nessa faixa etária, muitos indivíduos estão aposentados, o que pode ser evidenciado também nos pacientes estudados. 
Em contraponto, estudo realizado no Japão, com dados de pacientes submetidos a cirurgia cardíaca entre 2008 e 2010, evidenciou a ausência de significância estatística na associação de ISC com a idade $(p=0,73)$ e o sexo $(p=0,13)^{(10)}$.

Quanto à raça/cor, prevaleceu a parda, dado autodeclarado pelos pacientes e muito próprio de cada região. A Bahia está entre os estados brasileiros com maior concentração de população negra e mestiça. Além disso, grande parcela dessa população vive em condições socioeconômicas desfavoráveis, com maiores dificuldades de acesso às oportunidades sociais e consequentemente maiores limitações na atenção à saúde ${ }^{(11)}$.

Foram encontradas na literatura diferenças quanto à procedência dos pacientes. Neste estudo, a maioria dos pacientes residia no interior da Bahia, enquanto que, em pesquisa realizada em outro estado brasileiro, 66,6\% dos pacientes que desenvolveram ISC após a realização da cirurgia cardíaca moravam na capital ${ }^{(12)}$. Esta divergência pode ser justificada pela ausência de serviços de complexidade igual ou semelhante à do hospital campo da pesquisa, fora dos limites dos grandes centros urbanos, uma realidade comum nos estados da região Nordeste do Brasil. Dessa forma, a constituição de redes assistenciais regionais hierarquizadas para cirurgias cardíacas configura-se uma estratégia importante para reduzir as desigualdades no acesso à saúde entre as regiões brasileiras, garantir a qualidade do cuidado e otimizar recursos e custos nesse âmbito ${ }^{(13)}$.

As comorbidades mais prevalentes nos grupos estudados foram a HAS e o DM, sobressaindo-se a primeira, com altos índices tanto nos pacientes que desenvolveram ISC quanto nos que não desenvolveram. Reafirma esse dado, pesquisa realizada com a análise de 896 prontuários de pacientes submetidos a cirurgia cardíaca, na qual a HAS esteve presente em 80,9\% desses pacientes e o DM em $42,8 \%{ }^{(12)}$. Os pacientes idosos apresentam maior número de problemas relacionados às doenças crônicas não transmissíveis. Entre os problemas relacionados ao sistema cardiovascular, a hipertensão arterial é comum nesse grupo de pessoas.
O diagnóstico prévio de HAS é também a condição médica mais comum para o adiamento ou suspensão de uma cirurgia, porque importantes alterações hemodinâmicas podem ocorrer durante um procedimento cirúrgico, sendo mais pronunciadas nos pacientes com hipertensão arterial. Essa realidade vem sendo progressivamente repensada uma vez que o crescente conhecimento da fisiopatologia da hipertensão, da terapêutica anti-hipertensiva e o desenvolvimento de novos anestésicos e relaxantes musculares com efeitos hemodinâmicos mínimos, além de protocolos de controle da dor no período pós-operatório, têm contribuído para reduzir as complicações relacionadas com o perioperatório do paciente portador de $\mathrm{HAS}^{(14)}$.

Estudo realizado em 16 centros especializados em cirurgia cardiotorácica na Holanda verificou que, entre os fatores de risco para o desenvolvimento de ISC, o diabetes foi considerado uma das comorbidades mais relevantes, devido às complicações fisiopatológicas que ocorrem e dificultam o processo de cicatrização ${ }^{(14)}$. Uma meta-análise sobre diabetes e risco para ISC, considerando estudos de 1985 a 2015, evidenciou associação entre diabetes e ISC com odds ratio (OR) igual a 1,53, também sendo maior a associação desses com a cirurgia cardíaca $\mathrm{OR}=2,03$ em comparação com cirurgias de outros tipos $(p=0,001)$. Desse forma, esses resultados apoiam a consideração do diabetes como um fator de risco independente para ISC e afirmam que são necessários esforços contínuos para melhorar os resultados cirúrgicos para pacientes diabéticos ${ }^{(15)}$.

Os antecedentes clínicos observados em um estudo brasileiro realizado recentemente com pacientes submetidos a cirurgia cardíaca, durante o período de um ano, evidenciaram maior prevalência de HAS $(93,90 \%)$, DM (41,46\%), dislipidemia $(30,48 \%)$ e tabagismo $(23,17 \%)^{(9)}$.

Este estudo não encontrou relevância nos índices relacionados a nutrição. No entanto, estudo brasileiro recente identificou que ISC foi a principal causa de readmissão em 87,5\% dos pacientes submetidos à revascularização do miocárdio e em 12,5\% dos submetidos aos implantes de prótese valvar $(\mathrm{p}<0,001)$, e esteve associada às 
variáveis obesidade e dislipidemia ${ }^{(16)}$. Em contraposição, estudo de meta-análise, que associou o Índice de Massa Corpórea (IMC) - que reflete o estado de nutrição dos pacientes - com ISC afirmou não ter encontrado resultados significantes $^{(15)}$.

Todos os pacientes deste estudo, tanto os casos como os controles, tiveram tempo de internação pré-operatório maior que 24 horas. Um estudo realizado com 32.707 pacientes que foram submetidos a cirurgia cardíaca, 40\% hospitalizados antes da data da cirurgia e 60\% admitidos no mesmo dia da cirurgia, obteve como resultado que 6,7\% dos casos de infecção ocorreram nos pacientes admitidos no mesmo dia versus $8,7 \%$ nos pacientes hospitalizados há mais tempo $(\mathrm{p}<0001)$, e a mortalidade intra-hospitalar foi de $1,5 \%$ versus $2,8 \%(\mathrm{p}<0001)$, respectivamente ${ }^{(17)}$. Dessa forma, a relação entre a exposição, período de hospitalização e ISC foi estatisticamente comprovada. A hospitalização antes do dia da cirurgia foi relacionada ao aumento de 1,9 vezes com o risco de ocorrência de infecção, maior do que a de outras comorbidades, incluindo diabetes. Nesse mesmo estudo, foi verificado que a cirurgia de emergência e a transfusão sanguínea no intraoperatório foram as únicas variáveis que possuíam o risco de infecção maior ou semelhante, quando comparado ao tempo de hospitalização anterior ao dia do procedimento.

As cirurgias de urgência e emergência constituem um fator de risco para ocorrência de ISC, pois, muitas vezes, os pacientes apresentam instabilidade do quadro clínico e até mesmo risco de vida. Além disso, o tempo reduzido para a realização do procedimento anestésico-cirúrgico, frequentemente pode interferir no preparo pré-operatório, como na antissepsia da pele e antibióticoprofilaxia, bem como na alteração da técnica cirúrgica adotada, por exigir rápida organização, a fim de atender às prioridades do momento. Condições como choque, hipoxemia, hipotermia, transfusões sanguíneas e antibioticoprofilaxia inadequada, que corriqueiramente estão presentes nas situações de urgência e emergência, são fatores predisponentes à infecção ${ }^{(18)}$.
Neste estudo, no grupo de casos, 10,5\% dos pacientes precisaram submeter-se à cirurgia de forma emergencial, enquanto todas as cirurgias do grupo controle foram eletivas. Pôde-se inferir, com base nesses resultados, que as cirurgias de urgência/emergência estiveram diretamente relacionadas ao desenvolvimento de ISC nesses pacientes submetidos a cirurgia cardíaca.

Vale salientar que, neste estudo, o baixo percentual de pacientes que realizaram cirurgia de emergência deve-se ao fato de o hospital lócus do estudo não possuir serviço de emergência como porta de entrada, e os pacientes são admitidos via regulação de leitos, oriundos de outros serviços ou do ambulatório.

Os procedimentos cirúrgicos cardíacos, devido à necessidade de manipulação de órgãos nobres e delicados, exigem maior tempo de preparo, de cirurgia e de hospitalização. Esses aspectos aumentam o risco para complicações pós-operatórias, incluindo a ocorrência de ISC. Muitos são os fatores que podem influenciar no aumento do risco de ocorrência de ISC relacionados diretamente ao paciente, ao ambiente, à técnica e à assistência prestada ${ }^{(19)}$. Essas complicações, além de aumentarem o tempo de permanência do paciente no hospital, geram custos elevados para as instituições que, cada vez mais, estão adotando protocolos institucionais para uma cirurgia segura ${ }^{(20)}$.

O estudo encontrou, para ambos os grupos, caso e controle, tempos de realização de cirurgia semelhantes, não podendo inferir que esse fator tenha sido relevante para a ocorrência das ISC. Não foram encontrados muitos estudos que abordassem a relação desses fatores. No entanto, estudo com pacientes japoneses submetidos a cirurgia cardíaca encontrou significância $(p<0.0001)$ na associação entre eles ${ }^{(10)}$.

A classificação ASA vai de I a V, melhor a pior condição clínica, respectivamente, e VI para classificar o paciente em morte cerebral ${ }^{(8)}$. É o métodos mais utilizado para a avaliação clínica pré-operatória dos pacientes e apresenta-se como fator de risco para a ocorrência de ISC, isto é, quanto mais grave a condição clínica do paciente, provavelmente maior será a ocorrência 
de infecções, incluindo ISC ${ }^{(21)}$. Estudo realizado com pacientes japoneses submetidos a cirurgia cardíaca, observou que o tempo para realização da cirurgia e uma elevada pontuação ASA foram significativos em predizer o risco de ISC ${ }^{(10)}$. Neste estudo, a classificação ASA que prevaleceu entre os grupos, caso e controle, de pacientes submetidos a cirurgia cardíaca foi o ASA III, em que o paciente tem uma ou mais de uma doença sistêmica moderada ou grave com limitação funcional importante, como DM ou HAS mal controladas, doença pulmonar obstrutivo-crônica, obesidade mórbida, hepatite ativa, dependência alcoólica, marca-passo cardíaco implantado, redução moderada da fração de ejeção, IRC em diálise regular, prematuridade, história de infarto agudo do miocárdio há mais de 3 meses, acidente vascular cerebral, isquemia cerebral transitória ou stents coronarianos ${ }^{(8)}$.

Quanto à profilaxia e ao tratamento das ISCs, a administração dos antimicrobianos é consenso. Levando em consideração estudos e manuais ${ }^{(4,19)}$ que orientam o uso de antibióticos profiláticos em cirurgias nas diversas especialidades médicas, muitos serviços de saúde elaboram seus protocolos, adequando o uso às suas características e necessidades.

No geral, essas diretrizes sugerem que a profilaxia por até 48 horas pode ser adequada para procedimentos cardíacos com a finalidade de evitar ISC, devido à necessidade de realização de vários procedimentos invasivos secundários para o processo cirúrgico, tais como: circulação extracorpórea, uso de dispositivos invasivos venosos e urinários, transfusão sanguínea e outros ${ }^{(22-24)}$.

No tocante a essa questão, vale destacar que, neste estudo, a continuação da antibioticoprofilaxia pelo grupo controle, não acometido por ISC, correspondeu a 100\%, enquanto que entre os pacientes casos, que desenvolveram ISC, apenas 63,2\% a mantiveram; a outra parte fez uso dos antibióticos somente no período intraoperatório. Outros estudos evidenciam o mesmo descumprimento às orientações com relação ao uso de antibióticos no período perioperatório e analisam o impacto dessa ação ${ }^{(24-25)}$.

Estudo multicêntrico realizado no Brasil sobre os fatores relacionados à falta de adesão ao uso dos protocolos institucionais de antibioticoprofilaxia no período perioperatório concluiu que a maioria dos profissionais envolvidos no processo cirúrgico conhecia as diretrizes institucionais e referiu que os principais problemas para a falta de adesão ao uso adequado e preconizado era a falta de disciplina no centro cirúrgico e a não divulgação das diretrizes de uso de antibioticoprofilaxia $^{(25)}$. Dessa forma, sugere a busca para soluções inovadoras nesse quesito, uma vez que os métodos atuais convencionais de intervenção não estão produzindo os resultados desejados.

Desse modo, identificar os fatores de risco associados a um tipo infecção é bastante complexo, uma vez que é frequente a ocorrência simultânea de fatores que determinam a infecção. Nesse sentido, a prevenção efetiva dá-se por meio do controle desses fatores de risco concomitantemente.

O processo cirúrgico requer o trabalho harmonioso dos seus atores, neste caso, os profissionais da saúde, especialmente enfermeiros e médicos envolvidos, responsáveis pela realização da maioria de cuidados e assistência prestados aos pacientes cirúrgicos no período perioperatório, tornando a prática profissional um instrumento poderoso de qualidade e de segurança nesse âmbito.

Este estudo foi local, desenvolvido em apenas um serviço hospitalar, e seus resultados não podem ser extrapolados para outros serviços hospitalares. A principal limitação do estudo está relacionada à pequena amostra e à sua incapacidade de determinar estatisticamente a associação entre os fatores de risco e a ocorrência das ICS nessa população. Outras limitações estiveram relacionadas à dificuldade de acesso aos prontuários físicos, determinada pelas condições de armazenamento, organização e ausência ou inadequação dos registros. No entanto, foi possível mostrar características de risco importantes e semelhantes para grupos de pacientes expostos. Portanto, a implantação e o uso contínuo de um banco de dados abrangente das informações cirúrgicas, envolvendo o pré, o intra e o pós-operatório, poderão ampliar esses 
conhecimentos e subsidiar trabalhos futuros mais robustos, nas diversas especialidades cirúrgicas.

Pretende-se que este estudo contribua com o serviço de saúde e seus profissionais, a fim de subsidiar o conhecimento e as estratégias para reduzir os índices de morbidade e mortalidade relacionadas à ISC em cirurgias cardíacas, uma vez que este é um dos serviços de referência de cirurgias cardiológicas do Sistema Único de Saúde na região, além de ser um hospital de formação reconhecido para profissionais da área de saúde no âmbito nacional.

\section{Conclusão}

Os resultados demonstraram semelhanças nos fatores de risco que podem influenciar na ocorrência das ISC, tanto nos pacientes que desenvolveram essa infecção quanto nos que não a desenvolveram. Os resultados corroboram o explicitado na vasta literatura sobre os fatores de risco relacionados a essa complicação.

Conclui-se que os fatores de risco para infecção de sítio cirúrgico em pacientes submetidos a cirurgias cardíacas requerem dos profissionais envolvidos no processo maior controle para reduzir os índices dessas complicações.

$\mathrm{Na}$ caracterização dos pacientes submetidos a procedimentos cirúrgicos cardíacos no hospital estudado, tanto nos casos quanto nos controles, foi possível observar a presença de fatores de risco, como tempo de internação pré-operatório por mais de 24 horas, estado físico comprometido ( $A S A$ III), doenças crônicas, como HAS e DM, e uso descontínuo e não uniforme da antibioticoprofilaxia.

O elevado número de casos de ISC no pós-operatório de procedimentos cirúrgicos cardíacos e toda a caracterização descrita neste estudo exigem, por parte da equipe de saúde perioperatória do hospital, maior conhecimento e iniciativa para implementar medidas mais efetivas para o controle dos fatores de risco que podem estar relacionados ao desenvolvimento dessas infecções, identificando os problemas potenciais e agindo para garantir a segurança nos cuidados aos pacientes cirúrgicos e a redução da morbidade e mortalidade.

\section{Colaborações:}

1. concepção, projeto, análise e interpretação dos dados: Cláudia Silva Marinho Antunes Barros, Luana Stela de Araújo Castro e Marimeire Morais Conceição;

2. redação do artigo e revisão crítica relevante do conteúdo intelectual: Cláudia Silva Marinho Antunes Barros, Ana Lúcia Arcanjo Oliveira Cordeiro, Luana Stela de Araújo Castro, Marimeire Morais Conceição e Márcia Maria Carneiro Oliveira;

3. aprovação final da versão a ser publicada: Cláudia Silva Marinho Antunes Barros, Ana Lúcia Arcanjo Oliveira Cordeiro e Márcia Maria Carneiro Oliveira.

\section{Referências}

1. World Health Organization. Health care-associated infections fact sheet [Internet]. Geneva. 2015 [cited 2016 Mar 26]. Available from: http://www.who.int/ gpsc/country_work/gpsc_ccisc_fact_sheet_en.pdf

2. Brasil. Agência Nacional de Vigilância Sanitária. Critérios Diagnósticos de Infecção Relacionada à Assistência à Saúde [Internet]. 2a ed. Brasília; 2017 [cited 2018 Feb 17]. Available from: http:// www20.anvisa.gov.br/segurancadopaciente/ i mages/documentos/livros/Livro 2 CriteriosDiagnosticosIRASaude.pdf

3. Brasil. DATASUS - Tecnologia da Informação a Serviço do SUS [database on the internet]. Brasilia; 2016 [cited 2017 Mar 22]. Available from: http:// tabnet.datasus.gov.br/cgi/tabcgi.exe?sih/cnv/qiba. def

4. Centers for Disease Control and Prevention. The Hospital Infection Control Practices Advisory Committee [Internet]. Guideline for prevention of surgical site infection. Am j infect control. 1999 [cited 2016 Mar 27];27(2):247-78. Available from: http://www.cdc.gov/hicpac/pdf/SSIguidelines.pdf

5. Centre for Disease Control. Surgical Site Infection (SSI) Event. In: Procedure-associated. Module SSI [Internet]. Atlanta; 2018 [cited 2018 Mar 21]. Available from: https://www.cdc.gov/nhsn/pdfs/ pscmanual/9pscssicurrent.pdf 
6. Mocanu V, Buth KJ, Johnston LB, Davis I. The importance of continued quality improvement efforts in monitoring hospital-acquired infection rates: a cardiac surgery experience. Ann Thorac Surg [Internet]. 2015 [cited 2016 Sep 15];99:2061-9. Available from: http://www.annalsthoracicsurgery. org/article/S0003-4975(15)00129-0/pdf

7. Cristofolini M, Worlitzsch D, Wienke A, Silber RE, Borneff-Lipp M. Surgical site infections after coronary artery bypass graft surgery: incidence, perioperative hospital stay, readmissions, and revision surgeries. Infection [Internet]. 2012 [cited 2016 Mar 26];40(4):397404. Available from: https://link.springer.com/ content/pdf/10.1007\%2Fs15010-012-0275-0.pdf

8. American Society of Anesthesiologists. ASA Physical Status Classification System [Internet]. Washington (DC);2014[cited2016Mar26].Availablefrom:https:// www.asahq.org/resources/clinical-information/ asa-physical-status-classification-system\#

9. Caldeira CAV, Soares AJC. Perfil clínico e epidemiológico dos pacientes que realizaram cirurgia cardíaca no Hospital Sul Fluminense HUSF. Rev Saúde [Internet]. 2017 [cited 2017 Mar 21];8(1):3-7. Available from: file://C:/Users/marin/ Downloads/607-2395-7-PB\%20(1).pdf

10. Morikane K, Honda H, Yamagishi T, Suzuki S. Differences in risk factors associated with surgical site infections following two types of cardiac surgery in Japanese patients. J Hosp Infect [Internet]. 2015 [cited 2016 Mar 21];90(1):15-21. Available from: http://dx.doi.org/10.1016/j.jhin.2014.11.022

11. Brasil. Ministério da Saúde. Painel de Indicadores do SUS. Temático Saúde da População Negra [Internet]. 2016 abr [cited 2016 Mar 21];7(10):1-82. Available from: http://bvsms.saude.gov.br/bvs/publicacoes/ tematico_saude_populacao_negra_v._7.pdf

12. Magalhães MGPDA, Alves LMO, Alcantara LFDM, Bezerra SMMDS. Mediastinite pós-cirúrgica em um hospital cardiológico de Recife: contribuições para a assistência de enfermagem. Rev Esc Enf USP [Internet]. 2012 [cited 2016 Mar 26];46(4):865-71. Available from: http://www.scielo.br/pdf/reeusp/ v46n4/12.pdf

13. Viacava F, Porto S, Laguardia J, Moreira RS, Ugá MAD. Diferenças regionais no acesso a cirurgia cardiovascular no Brasil, 2002-2010. Ciênc Saúde Coletiva [Internet]. 2012 [cited 2016 Mar 21];17(11):2963-9. Available from: http://www.scielo.br/scielo.php?script=sci_ arttext\&pid $=$ S1413-81232012001100013\&lng $=$ pt\&t $\operatorname{lng}=\mathrm{pt}$
14. Feitosa ACR, Marques AC, Caramelli B,Ayub B, Polanczyk CA, Jardim C, et al. II Diretriz de Avaliação Perioperatória da Sociedade Brasileira de Cardiologia. Arq Bras Cardiol [Internet]. 2011 [cited 2016 Mar 21];96:1-68. Available from: http://www.scielo.br/scielo.php?script=sci_ arttext\&pid=S0066-782X2011000800001\&lng=pt\&n $\mathrm{rm}=\mathrm{iso} \& \ln \mathrm{g}=\mathrm{pt}$

15. Martin ET. Diabetes and risk of surgical site infection: a systematic review and meta-analysis. Infect Control Hosp Epidemiol [Internet]. 2016 Jan [cited 2017 Mar 21];37(1):88-99. Available from: https://www.ncbi.nlm.nih.gov/pmc/articles/ PMC4914132/pdf/nihms794109.pdf

16. Barreiros BRN, Bianchi ERF, Turrini RNT, Poveda VDB. Causas de readmissão hospitalar após cirurgia cardíaca. Rev Eletr Enferm [Internet]. 2016 [cited 2017 Mar 21];18:1-8. Available from: https:// revistas.ufg.br/fen/article/view/39529

17. Kelava M, Robich M, Houghtaling PL, Sabik JF, Gordon S, Mihaljevic T, et al. Hospitalization before surgery increases risk for postoperative infections. J Thorac Cardiovasc Surg [Internet]. 2014 [cited 2016 Mar 26];148(4):1615-21. Available from: http://dx.doi.org/10.1016/j.jtcvs.2014.06.067

18. Barbosa MH, Luiz RB, Andrade EV, Silva QCG, Mattia AL. Ocorrência de infecção de sítio cirúrgico em cirurgias de urgência e emergência. Rev Min Enferm. 2011 [cited 2016 Mar 21];15(34):254-8. Available from: file://C:/Users/marin/Downloads/ v15n2a14.pdf

19. Brasil. Agência Nacional de Vigilância Sanitária. Programa Nacional de Prevenção e Controle de Infecções Relacionadas à Assistência à Saúde (20162020) [Internet]. Brasília; 2016 nov [cited 2018 Apr 11]. Available from: http://portal.anvisa.gov.br/ documents/33852/3074175/PNPCIRAS+2016-2020/ f3eb5d51-616c-49fa-8003-0dcb8604e7d9

20. Beccaria LM, Cesarino CB, Werneck AL, Correio NCG, Correio KSS, Correio MNM. Complicações pós-operatórias em pacientes submetidos à cirurgia cardíaca em hospital de ensino. Arq Ciênc Saúde [Internet]. 2015 [cited 2017 Mar 26];22(3):3741. Available from: http://www.cienciasdasaude. famerp.br/index.php/racs/article/view/216/116

21. National Institute for Health and Care Excellence. Prevention and control of healthcare-associated infections overview [Internet]. London; 2017 [cited 2018 Mar 26]. Available from: file://C:/ Users/marin/Downloads/prevention-and-controlof-healthcare-associated-infections-prevention- 
and-control-of-healthcare-associated-infectionsoverview.pdf

22. Hamouda K, Oezkur M, Sinha B, Hain J, Menkel H, Leistner M, et al. Different duration strategies of perioperative antibiotic prophylaxis in adult patients undergoing cardiac surgery: An observational study. J Cardiothorac Surg [Internet]. 2015 [cited 2017 Mar 26];10(1):1-8. Available from: https://www.ncbi.nlm.nih.gov/pmc/articles/ PMC4345000/pdf/13019_2015_Article_225.pdf

23. Brasil. Agência Nacional de Vigilância Sanitária. Diretriz Nacional para Elaboração de Gerenciamento do Uso de Antimicrobianos em Serviços de Saúde [Internet]. Brasília; 2017 dez [cited 2018 Mar 27]. Available from: http:// portal.anvisa.gov.br/documents/33852/271855/ Diretriz+Nacional+para+Elaboração+de+Pro grama $+\mathrm{de}+$ Gerenciamento $+\mathrm{do}+\mathrm{Uso}+\mathrm{de}+\mathrm{An}$
timicrobianos+em+Serviços +de+Saúde/667 979c2-7edc-411b-a7e0-49a6448880d4

24. Leaper DJ, Edmiston CE. World Health Organization: global guidelines for the prevention of surgical site infection. J Hosp Infect [Internet]. 2017 Feb [cited 2018 Mar 16];95(2):135-6. Available from: http://linkinghub.elsevier.com/retrieve/pii/ S0195670116305874

25. Schmitt C, Lacerda RA. Fatores relacionados à adesão às recomendações para administração de redose em cirurgias limpas. Rev Eletr Enferm [Internet]. 2015 [cited 2016 Oct 9];17(1):78-84. Available from: http://revistas.ufg.br/index.php/ fen/article/view/22676

Recebido: 27 de março de 2018

Aprovado: 21 de agosto de 2018

Publicado: 13 de novembro de 2018

A Revista Baiana de Enfermagem utiliza a Licença Creative Commons - Atribuição-NãoComercial 4.0 Internacional.

https://creativecommons.org/licenses/by-nc/4.0/

Este artigo é de acesso aberto distribuído sob os termos da Licença Creative Commons (CC BY-NC).

Esta licença permite que outros remixem, adaptem e criem a partir do seu trabalho para fins não comerciais. Embora os novos trabalhos tenham de lhe atribuir o devido crédito e não possam ser usados para fins comerciais, os usuários não têm de licenciar esses trabalhos derivados sob os mesmos termos. 